\title{
Whitehead problems for words in $\mathbb{Z}^{m} \times F_{n}$
}

\author{
Jordi Delgado* \\ Dept. Mat. Apl. III \\ Universitat Politècnica de Catalunya, \\ Manresa, Barcelona \\ jorge.delgado@upc.edu \\ jdelgado. upc@gmail.com
}

January 15, 2013

\begin{abstract}
We solve the Whitehead problem for automorphisms, monomorphisms and endomorphisms in $\mathbb{Z}^{m} \times F_{n}$ after giving an explicit description of each of these families of transformations.
\end{abstract}

We generically call Whitehead problems for a finitely presented group $G$ the problems consisting in, given two objects (of the same certain suitable kind $\mathcal{O}$ ) in $G$, and a family $\mathcal{F}$ of transformations, decide whether there exists an element in $\mathcal{F}$ sending one object to the other. Specifically we will write $\operatorname{WhP}(\mathcal{O}, \mathcal{F})$ to mean the Whitehead problem with objects in $\mathcal{O}$ and transformations in $\mathcal{F}$, i.e.

$$
\left.\operatorname{WhP}(\mathcal{O}, \mathcal{F}) \equiv \dot{\iota} \exists \varphi \in \mathcal{F} \text { such that } o_{1} \stackrel{\varphi}{\mapsto} o_{2} ?_{\left(o_{1}, o_{2}\right.} \text { in } \mathcal{O}\right) .
$$

It is customary to include as a part of the problem, the search of one of such transformations, in case that there exists. So will we.

The kind of "objects in $G$ " usually considered include elements (i.e. words in the generators), subgroups, and conjugacy classes, as well as tuples of these; while the typical families of transformations (which we will always think acting on the right) are those of automorphisms, monomorphisms, epimorphisms and endomorphisms of $G$; we denote them respectively by Aut $G$, Mon $G$, Epi $G$ and End $G$.

*The author thanks the hospitality of the Centre de Recerca Matemàtica (CRMBarcelona) along the research programme on Automorphisms of Free Groups during which this preprint was finished; and gratefully acknowledge the support of Universitat Politècnica de Catalunya through the PhD grant number 81-727 and the MEC (Spain) through project number MTM2011-25955. 
This whole family of problems arise from the seminal $\operatorname{WhP}\left(F_{n}\right.$, Aut $\left.F_{n}\right)$, where $F_{n}$ denotes the free group on $n$ generators. It was proposed and solved by Whitehead in [6] using a (now classical) technique called peak-reduction.

In this note we will deal with Whitehead problems for words in finitely generated free-abelian times free groups (see [4] for full details). In sake of notational easiness we will hereafter usually abbreviate $G=\mathbb{Z}^{m} \times F_{n}$. Concretely we will solve $\operatorname{WhP}(G$, Aut $G), \operatorname{WhP}(G$, Mon $G)$ and $\operatorname{WhP}(G$, End $G)$. It is not surprising that the (already solved) corresponding problems for $\mathbb{Z}^{m}$ and $F_{n}$ emerge when considering Whitehead problems for $G$.

For the free-abelian groups the problems considered become those of the existence of solutions (of certain type) for integer matrix equations of the form $\mathbf{a} \cdot \mathbf{X}=\mathbf{b}$. This can be easily decided using linear algebra.

Proposition 1. Let $m \geqslant 1$, then

(i) $\operatorname{WhP}\left(\mathbb{Z}^{m}\right.$, Aut $\left.\mathbb{Z}^{m}\right)$ is solvable.

(ii) $\operatorname{WhP}\left(\mathbb{Z}^{m}\right.$, Mon $\left.\mathbb{Z}^{m}\right)$ is solvable.

(iii) $\operatorname{WhP}\left(\mathbb{Z}^{m}\right.$, End $\left.\mathbb{Z}^{m}\right)$ is solvable.

The same problems for the free group $F_{n}$ are much more complicated. As mentioned above, the case of automorphisms was already solved by Whitehead back in the 30's of the last century. The case of endomorphisms can be solved by writing a system of equations over $F_{n}$ (with unknowns being the images of a given free basis for $F_{n}$ ), and then solving it by the powerful Makanin's algorithm. Finally, the case of monomorphisms was recently solved by Ciobanu and Houcine.

Theorem 2. Let $n \geqslant 2$, then

(i) [Whitehead, [6]] $\mathrm{WhP}\left(F_{n}\right.$, Aut $\left.F_{n}\right)$ is solvable.

(ii) [Ciobanu-Houcine, [1]] $\mathrm{WhP}\left(F_{n}, \operatorname{Mon} F_{n}\right)$ is solvable.

(iii) [Makanin, [5]] $\mathrm{WhP}\left(F_{n}\right.$, End $\left.F_{n}\right)$ is solvable.

So, the auto, mono and endo Whitehead problems (for words) are solvable for both $\mathbb{Z}^{m}$ and $F_{n}$. For $G=\mathbb{Z}^{m} \times F_{n}$ though, these problems turn out to be more than the mere juxtaposition of the corresponding problems for its factors. That is because the endomorphisms of $G$ are more than pairs of endomorphisms of $\mathbb{Z}^{m}$ and $F_{n}$ as well. It is not difficult to obtain a complete description of them imposing the preservation of the (commutativity) relations defining $G$. 
Proposition 3. The endomorphisms of $G=\mathbb{Z}^{m} \times F_{n}$ are given by

$$
\Psi_{\phi, \mathbf{Q}, \mathbf{P}}:(\mathbf{a}, u) \mapsto\left(\mathbf{a Q}+\mathbf{u P}, u \phi_{\mathbf{a}}\right)
$$

where $\mathbf{u}=u^{\mathrm{ab}} \in \mathbb{Z}^{n}, \mathbf{Q}$ and $\mathbf{P}$ are integer matrices, and $\phi_{\mathbf{a}}: F_{n} \rightarrow F_{n}$ is either

(i) an endomorphism $\phi: F_{n} \rightarrow F_{n}$ (independent from $\mathbf{a}$ ), or

(ii) a map $u \mapsto w^{\alpha(\mathbf{a}, \mathbf{u})}$ where $w$ is a non-proper power in $F_{n} \backslash\{1\}$ and $\alpha(\mathbf{a}, \mathbf{u})=\mathbf{a l}^{\top}+\mathbf{u h}^{\top} \in \mathbb{Z}$ for certain $\mathbf{l} \in \mathbb{Z}^{m} \backslash\{\mathbf{0}\}$ and $\mathbf{h} \in \mathbb{Z}^{n}$.

We will refer to them as type(I) and type(II) endomorphisms of $G$ respectively.

Note that if $n=0$ then type (I) and type(II) endomorphisms do coincide. Otherwise, it turns out that type(II) endomorphisms are a sort of degenerated case corresponding to a free contribution from the abelian part while all the injective and exhaustive endomorphisms of $G$ are of type(I). Indeed, viewing $\mathbf{Q}$ as the endomorphism of $\mathbb{Z}^{m}$ given by right multiplying by $\mathbf{Q}$, we have the following quite natural characterization (note that the matrix $\mathbf{P}$ plays absolutely no role in this matter).

Proposition 4. Let $\Psi$ be an endomorphism of $G=\mathbb{Z}^{m} \times F_{n}$, with $n \geqslant 2$. Then,

(i) $\Psi$ is a monomorphism if and only if it is of type (I) with $\phi$ a monomorphism of $F_{n}$ and $\mathbf{Q}$ a monomorphism of $\mathbb{Z}^{m}$ (i.e. $\operatorname{det} \mathbf{Q} \neq 0$ ).

(ii) $\Psi$ is an epimorphism if and only if it is of type (I) with $\phi$ an epimorphism of $F_{n}$ and $\mathbf{Q}$ an epimorphism of $\mathbb{Z}^{m}$ (i.e. $\operatorname{det} \mathbf{Q}= \pm 1$ ).

The hopfianity of $\mathbb{Z}^{m}$ and $F_{n}$ together with this last proposition provide immediately the following results.

Corollary 5. $\mathbb{Z}^{m} \times F_{n}$ is hopfian and not cohopfian.

Corollary 6. An endomorphism of $G=\mathbb{Z}^{m} \times F_{n}(n \geqslant 2)$ is an automorphism if and only if it is of type (I) with $\phi \in \operatorname{Aut}\left(F_{n}\right)$ and $\mathbf{Q} \in \mathrm{GL}_{m}(\mathbb{Z})$.

Now we have the ingredients to prove the main result of this note.

Theorem 7. Let $G=\mathbb{Z}^{m} \times F_{n}$ with $m \geqslant 1$ and $n \geqslant 2$, then

(i) $\operatorname{WhP}(G$, Aut $G)$ is solvable.

(ii) $\operatorname{WhP}(G$, Mon $G)$ is solvable.

(iii) $\operatorname{WhP}(G$, End $G)$ is solvable. 
Sketch of the proof. We are given two elements $(\mathbf{a}, u),(\mathbf{b}, v) \in G$, and have to decide whether there exists an automorphism (resp. monomorphism, endomorphism) of $\mathbb{Z}^{m} \times F_{n}$ sending one to the other; and in affirmative case, find one of them.

Using the previous descriptions for each type of transformations in $\mathbb{Z}^{m} \times$ $F_{n}$ and separating the free-abelian and free parts, our problems reduce to deciding whether there exist integer matrices $\mathbf{P}, \mathbf{Q}$ and a transformation $\phi$ of $F_{n}$ ( $\mathbf{Q}$ and $\phi$ of certain kind depending on the case, see proposition 4) such that the two following independent conditions hold.

$$
\left.\begin{array}{rl}
u \phi & =v \\
\mathbf{a Q}+\mathbf{u P} & =\mathbf{b}
\end{array}\right\}
$$

Note that the subproblem associated to condition (1) becomes respectively the already solved $\operatorname{WhP}\left(F_{n}\right.$, Aut $\left.F_{n}\right), \operatorname{WhP}\left(F_{n}, \operatorname{Mon} F_{n}\right)$ and $\operatorname{WhP}\left(F_{n}\right.$, End $\left.F_{n}\right)$ in the cases of autos, monos, and endos of type (I), and is straightforward to check for endos of type (II). Thus, if there is not any $\phi$ solving each of these problems (for $F_{n}$ ), then the corresponding problem (for $G$ ) has no solution either, and we are done.

Otherwise, the decision method provides such a $\phi$ and our target reduces to solving the subproblem associated to condition (2): given arbitrary elements $\mathbf{a} \in \mathbb{Z}^{m}$ and $\mathbf{u} \in \mathbb{Z}^{n}$, decide whether there exist integer matrices $\mathbf{P}$ and $\mathbf{Q}$ (satisfying $\operatorname{det} \mathbf{Q} \neq 0$ in the case of monos, and $\operatorname{det} \mathbf{Q}= \pm 1$ in the case of autos) such that $\mathbf{a Q}+\mathbf{u P}=\mathbf{b}$.

If $\mathbf{a}=\mathbf{0}$ or $\mathbf{u}=\mathbf{0}$, these are well known results in linear algebra, otherwise write $\alpha=\operatorname{gcd}(\mathbf{a}) \neq 0$ and $\mu=\operatorname{gcd}(\mathbf{u}) \neq 0$. Then, the problems reduce to test whether the following linear system of equations

$$
\left.\begin{array}{cccc}
\alpha x_{1}+\mu y_{1} & = & b_{1} \\
& \vdots & \vdots & \\
\alpha x_{m} & +\mu y_{m} & = & b_{m}
\end{array}\right\}
$$

has integral solutions $x_{1}, \ldots, x_{m}, y_{1}, \ldots, y_{m} \in \mathbb{Z}$ (with no extra condition in the case of endos, satisfying $\left(x_{1}, \ldots, x_{m}\right) \neq \mathbf{0}$ in the case of monos, and satisfying $\operatorname{gcd}\left(x_{1}, \ldots, x_{m}\right)=1$ in the case of autos).

So, for the case of endos the decision is a standard argument in linear algebra. In the case of monomorphisms, the condition $\left(x_{1}, \ldots, x_{m}\right) \neq \mathbf{0}$ turns out to be superfluous and the same standard argument works, while the more involved case of autos become an exercise in elementary arithmetic and is decidable as well.

Finally, observe that in any of the affirmative cases, we can use the description in proposition 3 to reconstruct a transformation $\Psi$ (of the corresponding type) such that $(\mathbf{a}, u) \Psi=(\mathbf{b}, v)$. 
We note that, very recently, a new version of the classical peak-reduction theorem has been developed by M. Day [3] for an arbitrary partially commutative group (see also [2]). These techniques allow the author to solve the Whitehead problem for this kind of groups, in its variant relative to tuples of conjugacy classes and automorphisms. As far as we know, $\operatorname{WhP}(G, \operatorname{Mon} G)$ and $\operatorname{WhP}(G$, End $G)$ remain unsolved for a general partially commutative group $G$. Our theorem 7 is a small contribution into this direction, solving these problems for free-abelian times free groups in a direct and selfcontained form.

\section{References}

[1] Ciobanu, L., and Houcine, A. The monomorphism problem in free groups. Archiv der Mathematik 94, 5 (2010), 423-434.

[2] DAY, M. B. Peak reduction and finite presentations for automorphism groups of right-angled artin groups. Geometry $\&$ Topology 13 (Jan. 2009), 817-855.

[3] DAY, M. B. Full-featured peak reduction in right-angled artin groups. arXiv:1211.0078 (Oct. 2012).

[4] Delgado, J., And Ventura, E. Algorithmic problems for free-abelian times free groups. arXiv:1301.2355 (Jan. 2013).

[5] Makanin, G. Equations in free groups (russian). Izv. Akad. Nauk SSSR Ser. Mat. 46 (1982), 1190-1273.

[6] Whitehead, J. H. C. On equivalent sets of elements in a free group. The Annals of Mathematics 37, 4 (Oct. 1936), 782-800. ArticleType: research-article / Full publication date: Oct., 1936 / Copyright (C) 1936 Annals of Mathematics. 